\title{
Radio Tomographic Imaging for Ambient Assisted Living
}

\author{
Maurizio Bocca, Ossi Kaltiokallio, and Neal Patwari \\ SPAN Lab, ECE Department, The University of Utah \\ 50 S. Central Campus Drive, Salt Lake City, UT 84102, USA \\ maurizio.bocca@utah.edu, npatwari@ece.utah.edu
}

\begin{abstract}
Accurate localization of people in indoor and domestic environments is one of the key requirements for ambient assisted living (AAL) systems. This chapter describes how the received signal strength (RSS) measurements collected by a network of static radio transceivers can be used to localize people without requiring them to wear or carry any radio device. We describe a technique named radio tomographic imaging (RTI), which produces real-time images of the change in the radio propagation field of the monitored area caused by the presence of people. People's locations are inferred from the estimated RTI images. We show results from a long-term deployment in a typical single floor, one bedroom apartment. In order to deal with the dynamic nature of the domestic environment, we introduce methods to make the RTI system self-calibrating. Experimental results show that the average localization error of the system is $0.23 \mathrm{~m}$. Moreover, the system is capable of adapting to the changes in the indoor environment, achieving high localization accuracy over an extended period of time.
\end{abstract}

Keywords: Wireless Networks, Indoor Localization, Received Signal Strength, Radio Tomographic Imaging.

\section{Introduction}

For ambient-assisted living (AAL) and elderly care applications, accurate localization of people in indoor and domestic environments is one of the most important requirements. The location information can be used for multiple purporses, e.g., to monitor the daily activities and observe the tendencies of people, to alert caretakers and doctors in case of abnormal behavior of events, to automate lights, appliances and air conditioning systems in order to reduce the electricity consumption, etc. Indoor localization has received considerable attention in recent years from the research community, and different systems and sensing technologies have been applied in the context of AAL. In this chapter, we present our research on received signal strength (RSS) based device-free localization (DFL) and we show how it can be successfully applied in real-world scenarios for AAL and elderly-care applications.

In an RSS-based DFL system, multiple low-power, wireless transceivers are deployed in the area to be monitored as to form a mesh network. Each device 
broadcasts packets and stores the RSS of the packets received from all the other devices forming the network. When people are located or move in the environment, they modify the way the radio signals transmitted by the nodes propagate [1, 2], by shadowing [3], reflecting [4], diffracting [5], or scattering [6] a subset of their multipath components 1-9]. The effect of people on the wireless links is reflected in the RSS measurements collected by the nodes [10]. By knowing the position of the nodes, an RSS-based DFL system is capable of estimating the position of the people found in the monitored area by processing the changes over time of the RSS measurements of all the links of the network 11 17]. Since in this type of system the only source of information is the RSS provided by the radio module of the wireless transceivers, we refer to the transceivers as sensors, and to the network as a radio frequency (RF) sensor network [18].

In the context of AAL and elderly-care applications, DFL systems provide considerable advantages over other technologies. Unlike other systems, they accurately localize and track the people in the environment without having them to carry or wear any radio device or sensor. This feature makes these systems more suitable to monitor the activities of elder people without causing them physical discomfort or requiring them to remember each day to activate or wear these devices [19] (something particularly challenging for elder people affected by dementia or other neuro-degenerative diseases). Compared to video-camera systems [20], DFL systems do not raise the same privacy concerns, as they can not identify the person or recognize in detail what she is doing. DFL systems are also minimally invasive, since the small wireless sensors composing the network can be embedded in the walls of the house or into furniture, appliances, and other every day objects found in common domestic environments. Moreover, they can localize people also through-wall and in furnished environments which would be hard to cover with infrared motion detectors. Besides their limited invasiveness and flexibility, DFL systems are also considerably cheaper than e.g. ultra-wideband (UWB) radar devices.

Accurate indoor localization is even more challenging in the domestic environment. As people perform various activities during their every-day life, objects of various size, shape, and material are constantly moved, changing the propagation patterns of the radio signals. Thus, RSS-based systems have to monitor and dynamically adapt to these changes, providing high localization accuracy in the long-term. For systems using fingerprinting methods or statistical models of the relationship between distance and RSS [21 24], these sudden changes of the radio environment make sub-meter accuracy difficult to achieve and ultimately lead to the need of time-consuming recalibrations of the models. In addition, from a system perspective, the communication protocol run by the sensors composing the network has to be robust to sensors' failures, interference from overlapping wireless networks, and faulty links. Overall, the system must reliably provide its service over the long-term, without requiring manual re-configurations, recalibrations or even re-starts. 
In this chapter, we describe how the temporal changes in the radio propagation field of a wireless mesh network caused by the presence of people, measured by means of RSS, can be used to estimate their positions. Our discussion starts from the observation of how a person crossing a wireless link between two communicating RF sensors affects the RSS measured by the two devices. We show that the change in RSS depends on the frequency channel, and we provide a theoretical framework to combine the information collected on different frequency channels into a unique measurement for the link. The RSS measurements of all the links of the network are then processed in real-time to generate images of the changes in the propagation field of the monitored area - a process named radio tomographic imaging (RTI).

We present the experimental results obtained during a long-term deployment of a DFL system in an apartment. In this testbed, we apply methods to perform an on-line recalibration of the reference RSS of the links of the network. This allows separating the changes in the RSS introduced by the movements of people from the ones due to changes in the domestic environment. Besides achieving high localization accuracy over the entire length of the deployment, we show how the position estimates provided by the system can be processed in order to derive higher-level information about the daily activities and tendencies of the monitored people.

\subsection{Outline of the Chapter}

In Section 2, we first show how the RSS measured by two communicating sensors is affected by the presence of a person in the proximity of the link line, i.e., the straight imaginary line connecting the two devices. We then describe how, for the same transmitter and receiver pair, the change in RSS varies depending on the frequency channel. The remainder of the section presents the RTI process, in which the RSS measurements collected on all the links of the network on multiple frequency channels are combined to form images of the change in the propagation field due to the presence of people in the monitored area. We also introduce the methods that make our DFL system self-calibrating in order to achieve accurate localization over the long-term in highly dynamic indoor environments. The longterm deployment carried out in an apartment is described in Section 4.1 and the results are presented in Section 4.2 Section 5 concludes the chapter.

\section{Radio Tomographic Imaging}

\subsection{Link Line Crossing and Fade Level}

An RTI system uses the RSS measurements collected on the links of a wireless mesh network to localize and track people found in monitored area. In AAL applications, RTI has to be carried out in indoor environments where multiple objects and obstructions are normally found. Thus, in this type of environments, multipath propagation of the radio signals is predominant. The RSS measured 
at the receiving end of a link is the result of a phasor sum of the waves impinging on its antenna. The result depends on the position of the receiver and the center frequency of the radio signal. When the waves have the same phase, the phasor sum is constructive; when the waves have opposite phase, the phasor sum is destructive. For link $l$, the RSS measured on channel $c$ at time instant $k, r_{l, c}(k)$, can be modeled as:

$$
r_{l, c}(k)=P_{c}-L_{l, c}-S_{l, c}(k)+F_{l, c}(k)-\eta_{l, c}(k), \quad c \in \mathcal{F}
$$

where $P_{c}$ is the transmit power of the nodes, $L_{l, c}$ the large scale path loss, $S_{l, c}$ the shadowing loss, $F_{l, c}$ the fading gain (or fade level [25]), $\eta_{l, c}$ the measurement noise, and $\mathcal{F}=\{1, \ldots, C\}$ is the set of measured radio frequencies.

The RSS, which usually does not show consistent variations when the environment is stationary [26], is otherwise affected by the presence and movement of people in the proximity of the link line [10], i.e., the straight imaginary line connecting two communicating devices. The link line here defined differs from the definition of line-of-sight (LoS), which refers to an obstruction free direct path between the transmitter and receiver of the link. Figure 1 shows the RSS measurements collected on three different radio frequencies for the same link when this is crossed two times by a person. The dynamics of the RSS varies significantly depending on the considered frequency channel. Channel A (blue solid line) shows two consistent $(8 \mathrm{dBm})$ drops of the RSS when the person crosses the link line and no significant variation when the person is located far away from it. Channel B (red dashed-dotted line) shows a more consistent variation even when the person is located slightly away from the link line. However, the average RSS remains approximately the same even when the link line is crossed. Channel C (black dashed line) shows a very large variation of the RSS even when the person is very far from the link line. Moreover, when the person crosses the link line, the RSS tends to increase.

The relation between human-induced RSS changes and steady-state narrowband fading has been modeled in 25] by using the concept of fade level. The fade level of a link varies in between two extremes, namely an anti-fade state and a deep fade state. The RSS of a link in anti-fade is the result of constructive multipath interference. For such a link, $F_{l, c}$ in (1) is positive. When the link line is obstructed by a person, the RSS on average decreases. On the contrary, the RSS of a link in deep fade is the result of destructive multipath interference. For such a link, $F_{l, c}$ in (1) is negative. When the link line is obstructed by a person, the RSS on average increases. Since both the large scale path loss $L_{l, c}$ and the shadowing loss $S_{l, c}$ change very slowly with the center frequency and the frequency channels available with the ZigBee, 802.15.4-compliant nodes used in this work span over $80 \mathrm{MHz}$ in the $2.4 \mathrm{GHz}$ band, we assume that both do not depend on the measured frequency channel $c$. Thus, $F_{l, c}$ can be calculated as:

$$
F_{l, c}(k)=r_{l, c}(k)-P_{c}+\eta_{l, c}(k) .
$$

Due to the measurement noise, $\eta_{l, c}(k)$, we can not directly measure the fade level of a link. To estimate it, we use the average RSS, $\bar{r}_{l, c}$, measured in an 


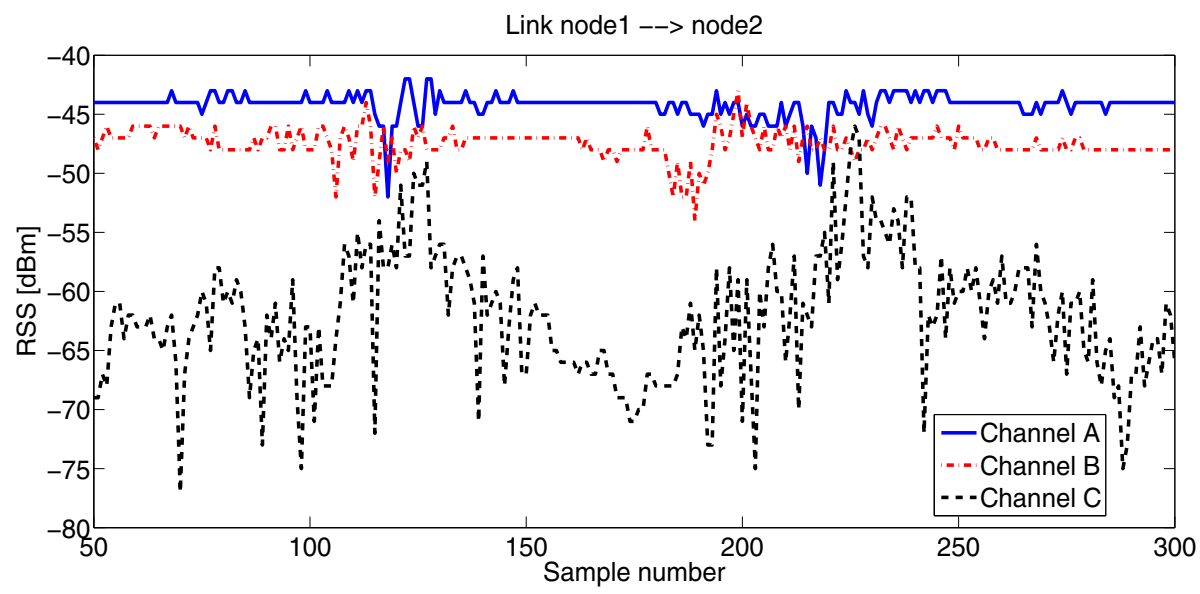

Fig. 1. The RSS measurements collected on three different radio frequencies for the same link when it is crossed two times by a person. The nodes are elevated $1 \mathrm{~m}$ from the floor and are $1.5 \mathrm{~m}$ apart. Channel A is ZigBee channel 15 (2425 MHz). Channel B is ZigBee channel $22(2460 \mathrm{MHz})$. Channel C is ZigBee channel $26(2480 \mathrm{MHz})$.

initial calibration of the system performed in stationary conditions, i.e. when the monitored area is empty. For each link $l$, the lowest $\bar{r}$ measured on the channels in $\mathcal{F}$ is used as a reference to derive the fade level during calibration $\bar{F}$ of channel $c$ :

$$
\bar{F}_{l, c}=\bar{r}_{l, c}-\min _{c} \bar{r}_{l, c} .
$$

A link is in a deeper fade on channel $c_{1}$ than on channel $c_{2}$ if $\bar{r}_{l, c_{1}}<\bar{r}_{l, c_{2}}$. Thus, in figure 1, channel A is the most anti-fade of the three, while channel $\mathrm{C}$ is the most deep fade. By definition, $\bar{F}_{l, c} \geq 0$ and $\bar{F}_{l, c}=0$ for one channel $c$ on each link.

For the purpose of localization, the difference between anti-fade and deep fade channels is substantial. As shown in Figure 1 for anti-fade channels, a person crossing the link line causes attenuation of the RSS, i.e., a sudden drop of several $\mathrm{dBm}$, which can be easily detected. Moreover, the area in which this change is measured is limited around the link line. Thus, anti-fade channels are the most informative to achieve an accurate localization. For deep fade links, the variation of the RSS is consistent even when the person is located at some position far away from the link line [16]. Thus, deep fade links are less suitable for accurate and timely detections of link line crossings, but can successfully be used to detect motion in the monitored environment, especially in sparse deployments, i.e. large areas covered by a small number of sensors. The difference between anti-fade and deep fade channels is illustrated also in Figure 3

The dynamics of the RSS measured on anti-fade and deep fade channels is affected also by the distance of the two communicating devices, as shown in Figure 2. As the distance becomes larger $(5 \mathrm{~m})$, the RSS drop measured on the anti-fade channel A (blue solid line) when the person crosses the link line 


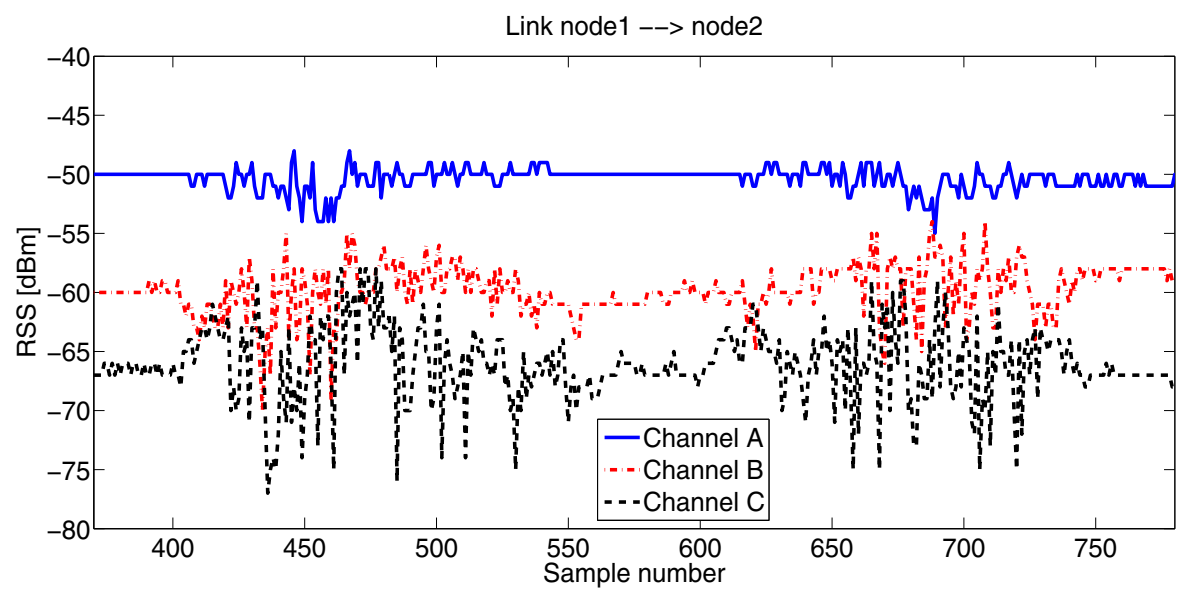

Fig. 2. The RSS measurements collected on three different radio frequencies for the same link when it is crossed two times by a person. The nodes are elevated $1 \mathrm{~m}$ from the floor and are $5 \mathrm{~m}$ apart. Channel A is ZigBee channel $15(2425 \mathrm{MHz})$. Channel B is ZigBee channel $22(2460 \mathrm{MHz})$. Channel C is ZigBee channel $26(2480 \mathrm{MHz})$.

is smaller $(4 \mathrm{dBm})$ and less predictable. Moreover, even the anti-fade channel measures a small variation of the RSS when the person is slightly far from the link line. Again, the channels having a deeper fade level pick up the presence of the person even when she is very far from the link line.

\subsection{Image Estimation}

An RTI system composed of $S$ static RF sensors deployed at known positions $\left\{\mathbf{z}_{s}\right\}_{s=1, \ldots, S}$ uses the RSS measurements $r_{l, c}(k)$, collected at time instant $k$ on all the $L$ links of the network on channel $c \in \mathcal{F}$, to estimate a discretized image of the change in the propagation field of the monitored environment, $\mathbf{x}$. The estimation problem can be modeled as:

$$
\mathbf{y}=\mathbf{W} \mathbf{x}+\mathbf{n},
$$

in which $\mathbf{y}$ and $\mathbf{n}$ are $L \times 1$ vectors representing the RSS measurements and noise of the $L$ links of the network, $\mathrm{x}$ is the $N \times 1$ discretized image to be estimated, where $N$ is the number of voxels of the image, and $\mathbf{W}$ is the $L \times N$ weight matrix, which tells how each voxel's attenuation impacts each link. Each element $x_{n}$ of $\mathbf{x}$ represents the change in the propagation field caused by the presence of a person in voxel $n$. The linear model for the change in the propagation field is based on the correlated shadowing models introduced in [11, 27] and the work in [12].

The change in RSS caused by the presence of people can be quantified using different methods. In attenuation-based RTI [12], the change in RSS is estimated as the difference between the RSS measured at time $k, r_{l, c}(k)$, and the average RSS measured during the initial calibration of the system, $\bar{r}_{l, c}$ : 


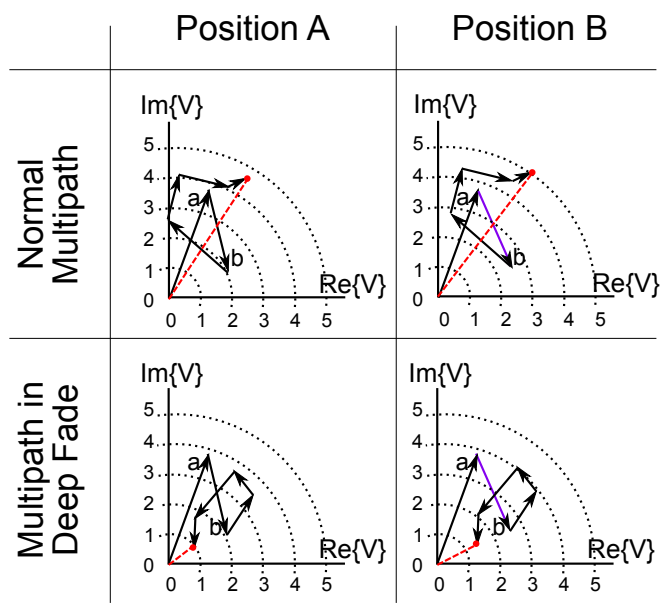

Fig. 3. Multipath contribute complex voltages to a phasor sum, the squared magnitude of which is the received power. Here, a person in Position A causes a frequency channel to have complex voltages as given in the left column. When the person moves to Position B (right column), they change path b by 10 degrees. For the normal multipath channel (top row) the multipath amplitude increases from 4.6 to 5.0, i.e., an increase of 0.7 $\mathrm{dB}$. For the channel in a deep fade, the same change in path b causes the amplitude to change from 1.0 to 1.5 , i.e., an increase of $3.5 \mathrm{~dB}$. Thus the same change is more noticeable when the channel's multipath are situated in a deep fade.

$$
\Delta r_{l, c}(k)=r_{l, c}(k)-\bar{r}_{l, c} .
$$

This method allows localizing both stationary and moving people, but requires an initial calibration of the system for the estimation of $\bar{r}_{l, c}$. In variance-based RTI [9], the change in RSS is quantified as the short-term unbiased sample variance of the RSS measurements:

$$
\hat{s}_{l, c}=\frac{1}{N_{s}-1} \sum_{p=0}^{N_{s}-1}\left(r_{l, c}(k-p)-\mu_{l, c}(k)\right)^{2},
$$

where:

$$
\mu_{l, c}(k)=\frac{1}{N_{s}} \sum_{p=0}^{N_{s}-1} r_{l, c}(k-p) .
$$

is the mean of the last $N_{s}$ RSS measurements of link $l$ on channel $c$. This method does not require an initial calibration of the system and can localize moving people, but is not capable of localizing stationary people. In histogram distance-based RTI [28], the change in RSS is quantified as the kernel distance [29] between the histogram of the most recently collected RSS measurements (the short-term histogram) and the histogram of RSS measurements collected during the calibration phase (the long-term histogram). Similarly to attenuation-based RTI, histogram 
distance-based RTI can localize both moving and stationary people, but also requires an initial calibration of the system.

Both attenuation-based RTI and histogram distance-based RTI are more suitable to AAL applications than variance-based RTI, since people living in a house can spend considerable amounts of time without moving, e.g., sleeping during the night or sitting on the couch. In this chapter, we consider only attenuationbased RTI and we introduce methods to make the system self-calibrating in order to achieve long-term accurate localization also in highly dynamic domestic environments.

For each link $l$, the RSS measurements collected on different frequency channels are combined into a unique RSS measurement $y_{l}$. As discussed in Section 2.1. anti-fade channels are more informative for localizing the people in the environment. In [16], the channels in $\mathcal{F}$ are ranked based on their fade level, from the most anti-fade to the most deep fade. If $\mathcal{A}_{i}$ is the set of size $m$ containing the indices of the $m$ top channels in the fade-level ranking, the link RSS measurement $y_{l}$ at time $k$ is calculated as:

$$
y_{l}(k)=\frac{1}{m} \sum_{c \in \mathcal{A}_{i}} \Delta r_{l, c}(k) .
$$

The results in [16] show that the optimal value of $m$, i.e. the number of channels considered in the computation of $y_{l}$, is different for each deployment of the RTI system. In the deployment described in this chapter, we include in the computation of $y_{l}$ the measurements collected on all the channels in $\mathcal{F}$, and we use the fade level $\bar{F}_{l, c}$ calculated as in (3) to weight them:

$$
y_{l}(k)=\frac{1}{\sum_{c \in \mathcal{F}} \bar{F}_{l, c}} \sum_{c \in \mathcal{F}} \bar{F}_{l, c} \cdot\left|\Delta r_{l, c}(k)\right| .
$$

The vector $\mathbf{y}$ is formed as follows:

$$
\mathbf{y}=\left[y_{1}, \ldots, y_{L}\right]^{T} .
$$

In RTI, the change in RSS measured on a link is assumed to be a spatial integral of the radio propagation field in the monitored area [1, 27]. Due to this, some voxels of the discretized image affect the RSS of a specific link, while some others do not. Each link's change in RSS is assumed to be a linear combination of the change in voxels' attenuation:

$$
y_{l}=\sum_{n=1}^{N} w_{l, n} x_{n}+\eta_{l},
$$

where $x_{n}$ is the change in attenuation of voxel $n, w_{l, n}$ the weight of voxel $n$ for link $l$, and $\eta_{l}$ the measurement noise of link $l$.

The weighting matrix $\mathbf{W}$ in (4) represents a spatial impact model between the $L$ links of the network and the $N$ voxels of the image. Each element $w_{l, n}$ of the matrix indicates how the change in RSS of voxel $n$ affects the RSS measurements 


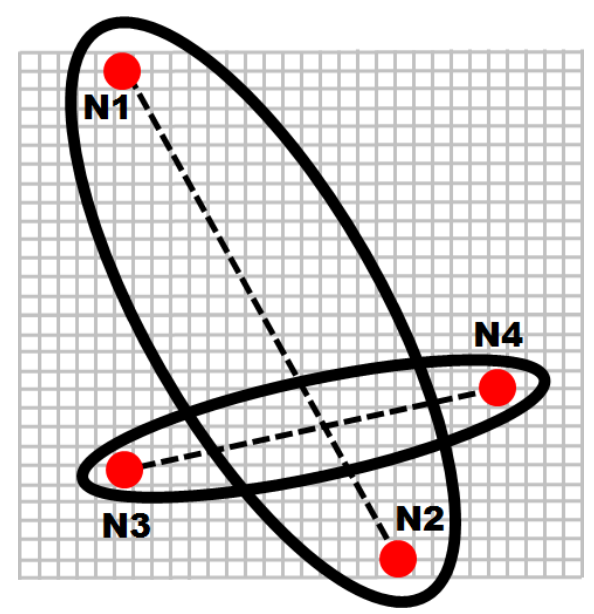

Fig. 4. The spatial impact model used in RTI is an ellipse having the foci located at the transmitter and receiver. The voxels located outside of the ellipse have their weight set to zero, while the voxels that are located within the ellipse have their weight set to a constant inversely proportional to the area of the ellipse. Thus, the shorter links (N3$\mathrm{N} 4$ ), which are more informative for localization, are weighted more than the longer ones (N1-N2).

of link $l$. The spatial model used in RTI [9, 11, 12, 16, 30, 31] is an ellipse having the foci located at the transmitter and receiver. According to this model, the voxels that are located outside of the ellipse have their weight set to zero, while the voxels that are located within the ellipse have their weight set to a constant which is inversely proportional to the area $\Gamma_{l}$ of the ellipse:

$$
w_{l n}= \begin{cases}\frac{1}{\Gamma_{l}} & \text { if } d_{l, n}^{t x}+d_{l, n}^{r x}<d_{l}+\lambda \\ 0 & \text { otherwise }\end{cases}
$$

where $d$ is the distance between the transmitter and receiver, $d_{l n}^{t x}$ and $d_{l n}^{r x}$ are the distances from the center of voxel $n$ to the transmitter and receiver of link $l$, respectively, and $\lambda$ is the excess path length of the ellipse, i.e., the parameter defining the width of the ellipse. In Section 2.1, we discussed how the shorter links are more informative for localization. In (12), by using a constant inversely proportional to the area of the ellipse, the shorter links are weighted more than the longer ones. The spatial impact model used in RTI is shown in Figure 4 ,

Since the number of links is considerably smaller than the number of voxels, estimating the image vector $\mathbf{x}$ is an ill-posed inverse problem, which requires regularization 32]. In this work, we use a regularized least-squares approach 11, 16, 30, 31]. The estimated image of the change in the propagation field is calculated as:

$$
\hat{\mathbf{x}}=\Pi \mathbf{y}
$$


where the inversion matrix is:

$$
\boldsymbol{\Pi}=\left(\mathbf{W}^{T} \mathbf{W}+\mathbf{C}_{x}^{-1} \sigma_{N}^{2}\right)^{-1} \mathbf{W}^{T},
$$

in which $\sigma_{N}^{2}$ is the regularization parameter. The a priori covariance matrix $\mathbf{C}_{x}$ is calculated by using an exponential spatial decay:

$$
\left[\mathbf{C}_{x}\right]_{j, i}=\sigma_{x}^{2} e^{-d_{j, i} / \delta_{c}},
$$

where $\sigma_{x}^{2}$ is the variance of voxel measurements, $d_{j, i}$ is the distance from the center of voxel $j$ to the center of voxel $i$, and $\delta_{c}$ is the voxels' correlation distance. The linear transformation $\boldsymbol{\Pi}$ is computed only once before real-time operation. The calculation of $\hat{\mathbf{x}}$ in (13) requires $L \times N$ operations and can be performed in real-time.

When only one person is in the monitored environment, her position $\hat{p}$ is estimated as:

$$
\hat{p}=\arg \max _{n \in N} \hat{\mathbf{x}},
$$

i.e., the person's position estimate is at the voxel $n$ of the discretized image $\hat{\mathbf{x}}$ having the highest value. In [9, 15, 31], the estimated trajectory followed by the person is smoothed by recursively applying a Kalman filter [33] on the position estimates. In this chapter, we present results related only to the localization of one person. For multiple people localization and tracking with RTI, the reader is invited to refer to the works in [17, 25, 34, 35].

\subsection{On-line Calibration for AAL Applications}

One of the main challenges in using an RTI system in real-world indoor and domestic environments is represented by the fact that they are highly dynamic, i.e., people working or living in these environments constantly change the position of objects of various size, shape and material while carrying out their activities. This changes dramatically over time the reference RSS of the links on the measured frequency channels, i.e. the RSS measured in stationary conditions. Figure 5 provides an example of the effect of environmental changes on the reference RSS: a person moves towards the link line carrying a metallic chair with her, then places the chair in the middle point of the link and moves away from the link line. The new position of the chair in the environment changes dramatically the RSS measured on the three different channels: for channel A (blue solid line), the reference RSS drops by $6 \mathrm{dBm}$; for channel B (red dashed-dotted line), the reference RSS drops by $5 \mathrm{dBm}$; for channel C (black dashed line), the reference RSS drops by $10 \mathrm{dBm}$.

By affecting the reference RSS, environmental changes modify also the fade level of the frequency channels. Before the chair is placed in the middle point of the link, channel $\mathrm{C}$ is the one in the deepest fade; after, channel $\mathrm{B}$ is the one in the deepest fade. Consequently, the fade level-based ranking of the channels derived during the initial calibration of the system which is used in [16] can be drastically modified even by environmental changes. In this work, the fade level 


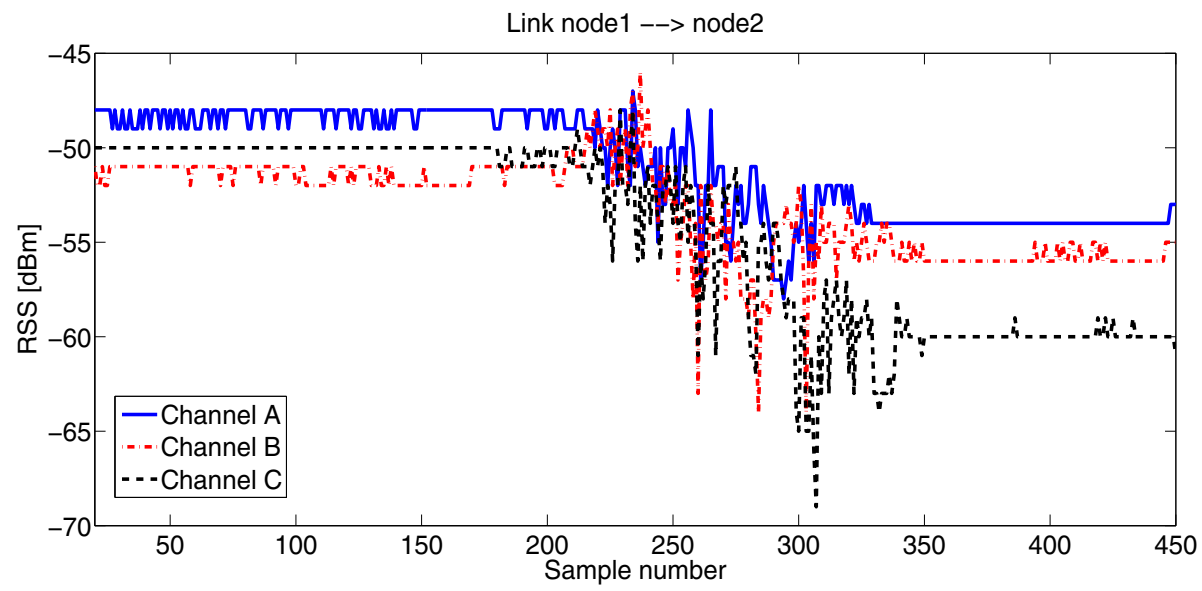

Fig. 5. The effect of environmental changes on the RSS measured on different radio frequencies for the same link. The nodes are elevated $1 \mathrm{~m}$ from the floor and are $4 \mathrm{~m}$ apart. At sample 200, a person carrying a chair starts moving towards the link line and places the chair in the middle point of the link. At sample 300, the person moves away from the link line leaving the chair behind. The presence of the chair on the link line changes considerably the RSS measured on the three channels.

is used in (9) to weight the RSS measurements of all the channels in $\mathcal{F}$. Thus, environmental changes modify also the weight assigned to the RSS measurements of the different channels.

These observations require methods to make the RTI system able to adapt to the changes in the propagation patterns of the radio signals and recalibrate on-line both the reference RSS and fade level of the frequency channels. Without recalibration, the RTI system would not be able to achieve accurate localization over an extended period of time and would need to be stopped, recalibrated and restarted frequently. In [9, 30, 36, 37], different methods to adapt to the dynamic environment are presented. In [30], the reference RSS $\bar{r}_{l, c}(k)$ of link $l$ on channel $c$ at time $k$ is calculated using a moving average:

$$
\bar{r}_{l, c}(k)=(1-\alpha) \bar{r}_{l, c}(k-1)+\alpha r_{l, c}(k),
$$

where $\alpha \in[0,1]$ is a parameter defining the rate of adaptation of the reference RSS, i.e., slow for low values, e.g., $\alpha=0.01$, fast for higher values, e.g., $\alpha=0.2$. In this work, we extend the on-line recalibration of the system to the fade level of the channels. The fade level $\bar{F}_{l, c}(k)$ of link $l$ on channel $c$ at time $k$ is calculated using the reference RSS calculated in (17):

$$
\bar{F}_{l, c}(k)=\bar{r}_{l, c}(k)-\min _{c} \bar{r}_{l, c}(k) .
$$

Consequently, at time $k$, the link measurement $y_{l}$ becomes:

$$
y_{l}(k)=\frac{1}{\sum_{c \in \mathcal{F}} \bar{F}_{l, c}(k)} \sum_{c \in \mathcal{F}} \bar{F}_{l, c}(k) \cdot\left|\Delta r_{l, c}(k)\right|
$$




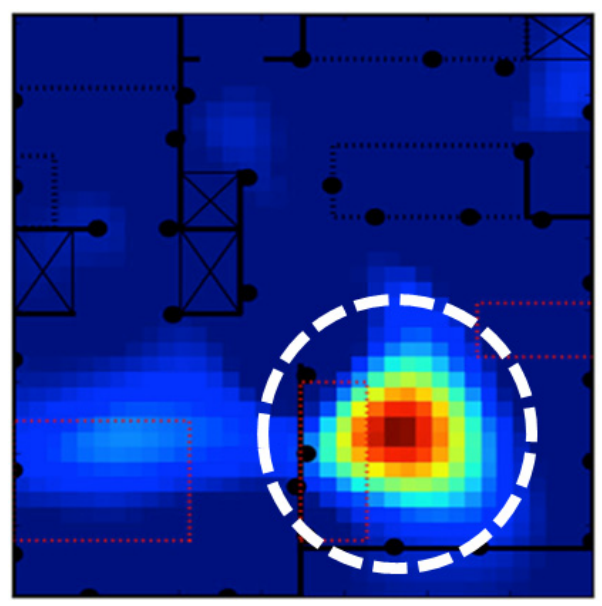

Fig. 6. A typical RTI image produced by the RTI system. The red blob indicates the area occupied by the person. The white dashed circle, centered at the current position estimate, represents the gating area outside of which the links are recalibrated on-line.

where:

$$
\Delta r_{l, c}(k)=r_{l, c}(k)-\bar{r}_{l, c}(k) .
$$

Figure 6 shows a typical RTI image produced by the system. The red blob in it indicates the area in which the person is located. When the person is stationary, the RSS measurements of the links intersecting the area occupied by the person are approximately constant. Due to the on-line recalibration of the reference RSS, if the person will not move for an extended period of time, e.g., while sleeping or sitting on the sofa, the RSS attenuation on those links, $\Delta r_{l, c}(k)$, will be very small, since the on-line reference $\mathrm{RSS}, \bar{r}_{l, c}(k)$, will have a value very close to the current RSS measurements. Thus, the link measurement $y_{l}$ will also have a very small value and the red blob indicating the position of the person will vanish in the background, making the position estimate $\hat{p}$ calculated in (16) noisy and unreliable.

To avoid the disappearing of the blob when the person is stationary for an extended period of time, we center a circular gating area of radius $\omega$ at the current position estimate $\hat{p}(k)$. The gating area is used as a spatial filter for the on-line recalibration of the reference RSS and fade level of the channels, as follows:

$$
\bar{r}_{l, c}(k)=\left\{\begin{array}{ll}
(1-\alpha) \bar{r}_{l, c}(k-1)+\alpha r_{l, c}(k) & \text { if } l \notin \mathcal{P}_{l} \\
\bar{r}_{l, c}(k-1) & \text { otherwise }
\end{array},\right.
$$

and similarly for the fade level of the channels:

$$
\bar{F}_{l, c}(k)=\left\{\begin{array}{ll}
\bar{r}_{l, c}(k)-\min _{c} \bar{r}_{l, c}(k) & \text { if } l \notin \mathcal{P}_{l} \\
\bar{F}_{l, c}(k-1) & \text { otherwise }
\end{array},\right.
$$


Table 1. Image reconstruction parameters

\begin{tabular}{lcc}
\hline \hline Description & Parameter & Value \\
\hline Pixel width [m] & $p$ & 0.20 \\
Ellipse excess path length[m] & $\lambda$ & 0.02 \\
Voxels standard deviation [dB] & $\sigma_{x}$ & 0.2236 \\
Noise standard deviation [dB] & $\sigma_{N}$ & 1 \\
Correlation coefficient & $\delta_{c}$ & 3 \\
Moving average coefficient & $\alpha$ & 0.05 \\
Gating area radius [m] & $\omega$ & 1 \\
\hline
\end{tabular}

where $\mathcal{P}_{l}$ is the set of links of the network intersecting the circle of radius $\omega$ centered at the current position estimate $\hat{p}(k)$. Thus, the links of the network not intersecting the gating area are recalibrated on-line, while the ones intersecting the gating area are not recalibrated. Thanks to this spatial filter, the red blob does not disappear from the image and the position estimate $\hat{p}$ remains on the spot occupied by the person even when she is stationary for an extended period of time. The values of the parameters used in methods described in this Section are listed in Table 1 .

\section{Hardware and Communication Protocol}

In this section, we describe the RF sensors composing the RTI system and the communication protocol used to collect the RSS measurements of all the links of the nework on multiple frequency channels.

\subsection{Hardware}

The experiments described in this chapter are carried out with Texas Instruments CC2531 USB dongle nodes [38], shown in Figure 7 The nodes are equipped with a low-power, 802.15.4 39] compliant radio operating in the $2.4 \mathrm{GHz}$ ISM band. In the experiments, we set the transmit power of the nodes to the maximum nominal value, i.e., $4.5 \mathrm{dBm}$.

The 802.15.4 standard defines 16 frequency channels, $5 \mathrm{MHz}$ apart and having $2 \mathrm{MHz}$ of bandwidth. The carrier frequency (in $\mathrm{MHz}$ ) of channel $c$ is:

$$
f_{c}=2405+5(c-11), c \in[11,26] .
$$

Due in part to the differences in antenna impedance matching across an $80 \mathrm{MHz}$ frequency band [40], the CC2531 nodes measure lower RSS values on the lower frequency channels than on the higher ones. Thus, to avoid bias in estimating the fade level of the channels, normalization is required. We experimentally derived the linear relationship $P_{c}=0.145 c+1.733$, witch closely matches the measured transmit power. 

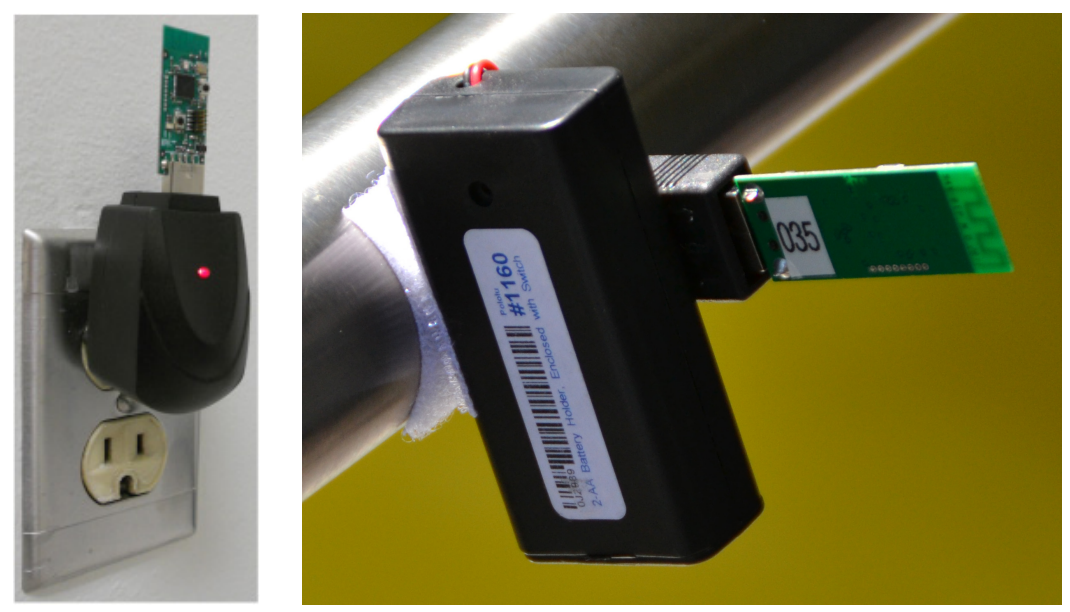

Fig. 7. Two figures of the CC2531 USB dongle node used in the experiments. On the left, a node plugged into an electricity socket through an USB power adapter. On the right, a node powered by two AA batteries.

In [30], we had noticed that the propagation pattern of the antenna of the nodes was heavily affected by the proximity to metallic surfaces and walls. This made the RSS measurements more noisy and decreased the localization accuracy. The new battery pack, visible in Figure 7 is now designed such that the antenna is pointing away from the surface or wall to which it is attached through velcro. This compact and lightweight design allows us reducing the time required to deploy the nodes and increase the quality of the RSS measurements.

\subsection{Communication Protocol: multi-Spin}

The RF sensors composing the RTI system run multi-Spin, a multi-channel TDMA protocol which defines the order of transmission of the nodes and synchronizes their switching on different frequency channels. In multi-Spin, time is divided into slots, cycles and rounds: a round includes $|\mathcal{F}|$ TDMA cycles; a TDMA cycle includes $S$ slots. In each slot, only one of the nodes transmits a packet, while all the other nodes are in receiving mode. The order of transmission of the nodes in a TDMA cycle is based on their built-in ID number. The set of measured frequency channels $\mathcal{F}$ is pre-defined by the user before deployment and stored as a list in the memory of the nodes.

In each cycle, the nodes communicate on one of the frequency channels of the list. At the end of the cycle, they switch synchronously on the next channel of the list. The first channel in the list acts as a backbone channel: if a node does not receive any packet for $S$ consecutive slots, it will assume to have lost synchronization with the network and will immediately switch back to the backbone channel, waiting for packets. In this way, when the other nodes will switch 


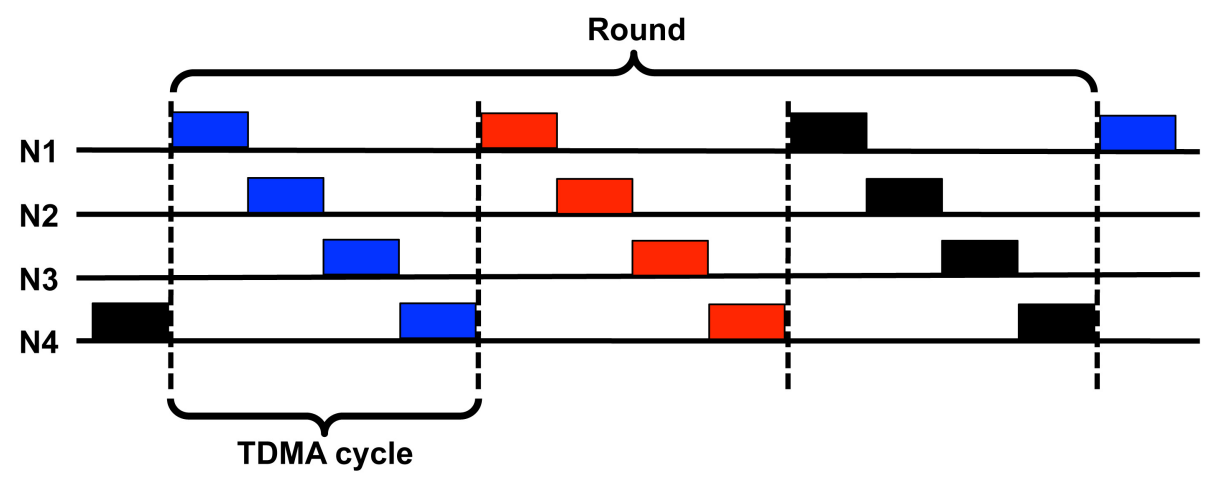

Fig. 8. In multi-Spin, time is divided into slots, cycles and rounds: a round includes $|\mathcal{F}|$ TDMA cycles; a TDMA cycle includes $S$ slots. In each slot, only one of the nodes of the network is transmitting (solid block), while the others are in receiving mode. The order of transmission is based on the built-in ID number of the nodes. At the end of a TDMA cycle, the nodes switch synchronously on the next frequency channel in the list pre-defined by the user.

again to the first channel of the list, the node previously fallen out-of-sync will be able to rejoin the network. This mechanism ensures that even when one or more nodes stop receiving packets from the others, e.g., because they have run out of power or have accidentally been unplugged from the electric socket, they will be able to rejoin the network without requiring a manual restart by the user.

multi-Spin starts when at least two nodes are turned on (if battery-powered) or plugged into electric sockets. Each node repeatedly calculates a random backoff time and transmits a packet on the backbone frequency channel until it receives a packet from another node. The reception of the first packet allows the nodes to synchronize themselves and communicate on the frequency channels of the list.

The payload of each packet includes the ID number of the transmitting node and the most recent RSS measurements of the packets received from the other nodes. A node connected to a laptop switches channels synchronously with the network of RF sensors and overhears all the traffic, collecting and processing in real-time the RSS measurements of all the links. By knowing the total number of sensors $S$ composing the network and the ID number of the transmitting node $I D_{T X}$, at the reception of a packet each node is able to calculate the number of slots until the next switching of frequency channel, $\Delta_{c}$, and the number of slots until the next transmission, $\Delta_{T X}$, as follows:

$$
\begin{gathered}
\Delta_{c}=S-I D_{T X}, \\
\Delta_{T X}= \begin{cases}I D_{R X}-I D_{T X}-1 & \text { if } I D_{T X}<I D_{R X} \\
S-I D_{T X}+I D_{R X} & \text { otherwise }\end{cases}
\end{gathered}
$$


where $I D_{R X}$ is the ID number of the receiving node. This mechanism makes the network tolerant to packet drops, due e.g. to the interference of other coexisting wireless networks [41, 42], as the nodes can keep on communicating and on being synchronized even when dropping packets.

The features described above make multi-Spin an autonomously starting, synchronizing and healing communication protocol tolerant to interference from coexisting wireless networks. With the CC2531 nodes, the average time length of a slot is approximately $3 \mathrm{~ms}$. For a network composed of $33 \mathrm{RF}$ sensors communicating on five frequency channels, such as the one described in Section 4.1, the total length of a TDMA cycle is approximately $100 \mathrm{~ms}$, making the total length of a round approximately equal to $500 \mathrm{~ms}$. Thus, an RTI system composed of 33 nodes collects two RSS measurements per link per second.

\section{Experimental Results}

\subsection{Deployment}

To evaluate the performance of the methods described in Section 2, we use the data collected during a three months deployment of an RTI system composed of 33 nodes in a typical $58 \mathrm{~m}^{2}$ single floor, one bedroom apartment inhabited by a single person. The nodes are set to communicate on five frequency channels, i.e., $\mathcal{F}=\{11,15,18,21,26\}$. The blueprint and an image of the apartment are shown in Figure 9

\subsection{Localization Accuracy}

In the beginning of the deployment, we evaluate the accuracy of the RTI system in localizing the person in different areas of the apartment. We define 14 spots of evaluation, in which the person stands without moving for a pre-determined amount of time before walking to the next position. Figure 10 shows the average position estimates provided by the RTI system in each of the 14 points of evaluation. The average localization error is $0.23 \mathrm{~m}$. It has to be noted that the localization error remains below $0.40 \mathrm{~m}$ in 13 of the 14 points of evaluation. The largest error, $0.92 \mathrm{~m}$, is measured when the person stands in the kitchen, where a large marble counter (visible also in Figure 9) has a remarkable impact on the propagation of the radio signals.

The effect of the on-line recalibration of the reference RSS and fade level of the measured frequency channels is shown in Figure11, The RTI images (a) and (b) are formed using the on-line recalibration methods described in Section 2.3. while the images (c) and (d) are formed using the reference RSS and fade levels estimated during the initial calibration of the system. Images (a) and (c) are formed six hours after the system is started $(\Delta T=6 \mathrm{~h}$ ), while images (b) and (d) are formed 18 hours after the system is started $(\Delta T=18 \mathrm{~h})$. Without on-line recalibration, the images formed by the RTI system rapidly become more noisy: while in image (a) the image shows only one clear blob in the position occupied 

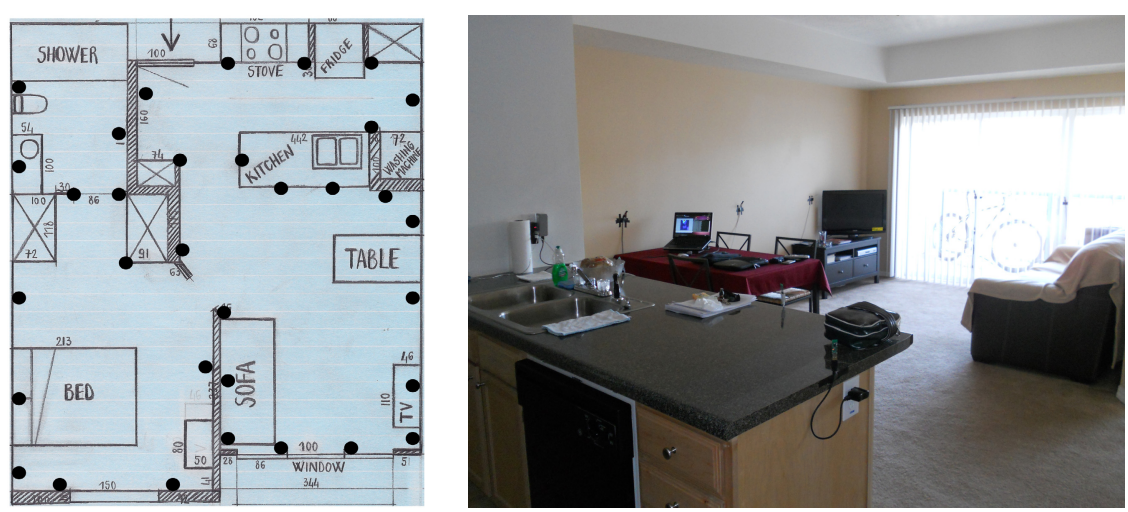

Fig. 9. On the left, the blueprint of the single floor, one bedroom apartment in which the RTI system has been deployed for over three months. The black dots represent the position of the $33 \mathrm{RF}$ sensors. On the right, an image of the apartment.

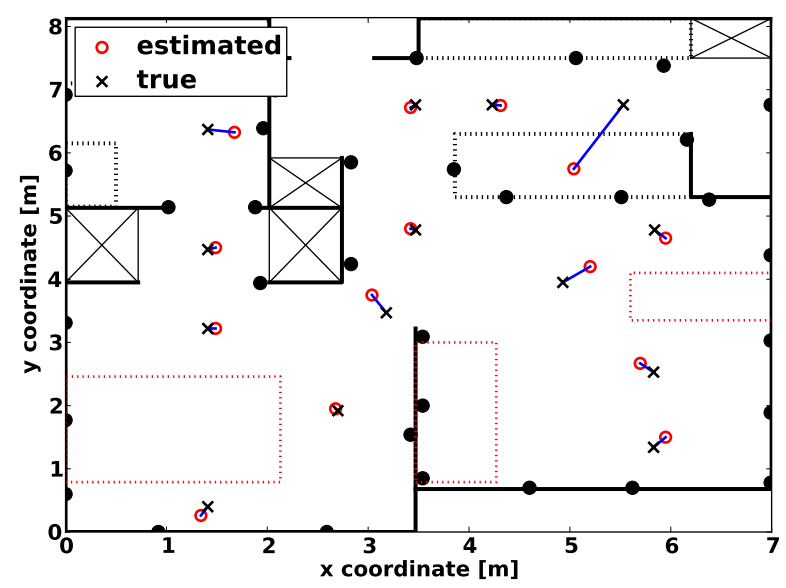

Fig. 10. The true and estimated position of the person in 14 different points of the apartment. The average localization error is $0.23 \mathrm{~m}$.

by the person, image (c) shows other three small blobs due to changes in the environment. After 18 hours, the effect of the on-line recalibration is even more evident: in image (b), the system can still correctly localize the person, forming an image that shows only one clear blob in the correct position. Without online recalibration (image(d)), the system forms a very noisy image with multiple blobs and provides a position estimate very far from the true location of the person. We invite the reader to view a video showing the movements of the person living in the apartment over a ten minutes time interval at [43]. 


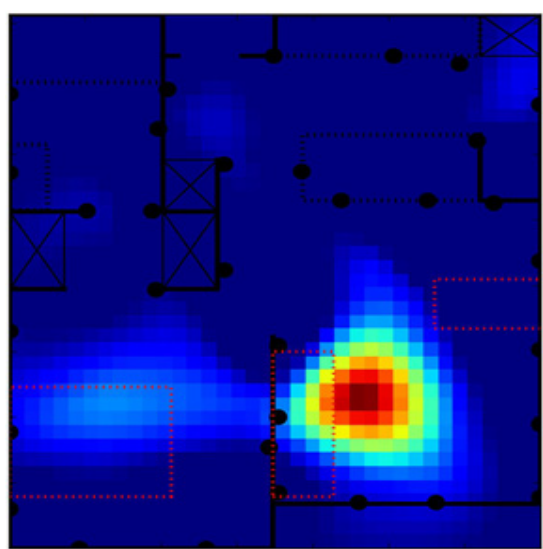

(a) $\Delta T=6 \mathrm{~h}$

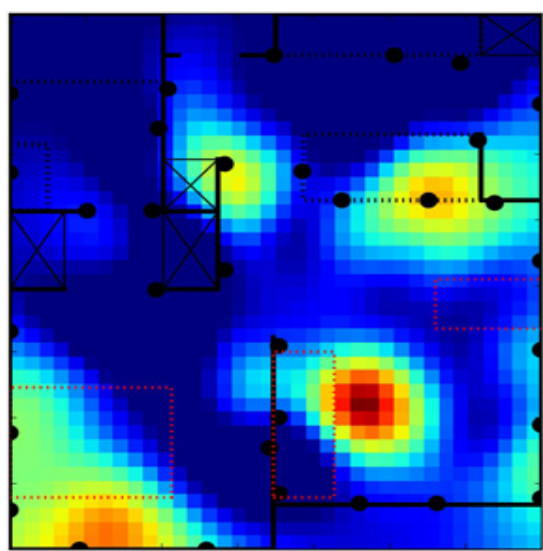

(c) $\Delta T=6 \mathrm{~h}$

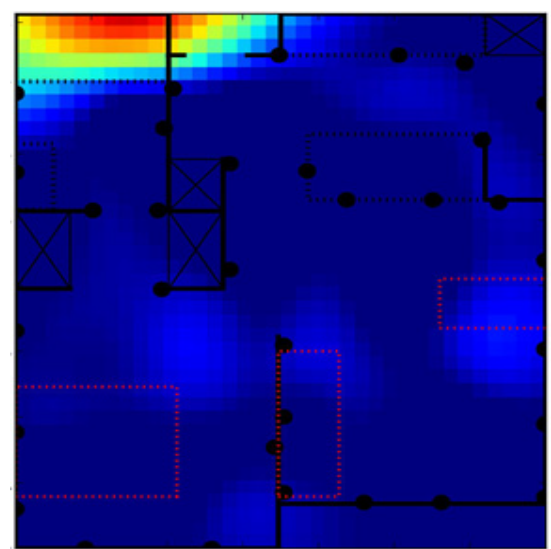

(b) $\Delta T=18 \mathrm{~h}$

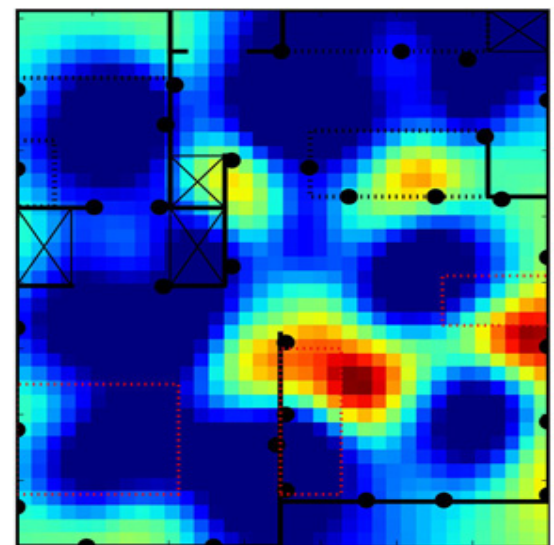

(d) $\Delta T=18 \mathrm{~h}$

Fig. 11. The effect of the on-line recalibration of the reference RSS and fade level of the measured frequency channels on the long-term localization accuracy. Without on-line recalibration (images (c) and (d)), the RTI images formed by the system rapidly become more noisy and ultimately lead to position estimates far from the true location of the person. On the other hand, a system using the on-line recalibration methods described in Section 2.3 can maintain high localization accuracy over an extended period of time despite the several environmental changes due to the daily activities carried out by the monitored person.

The location information can be used in AAL applications to infer about the daily routine and the health of the monitored person. The apartment can be divided into areas-of-interest (AoI), e.g., the kitchen, bathroom, bedroom, living room, etc. The highly accurate localization provided by an RTI system allows further dividing each AoI into smaller sub-areas which can be associated to specific activities, e.g., cooking on the stove, washing laundry, taking a shower, 


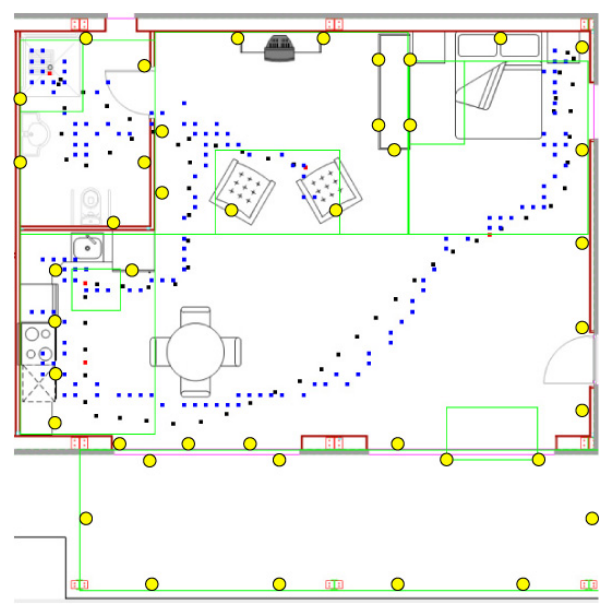

Fig. 12. The blueprint of the home in which the tests of the second $E v A A L$ competition were carried out. The yellow circles represent the RF sensors. The black dots represent the true position of the person during the test at $1 \mathrm{~s}$ intervals. The red dots represent the true position of the person at spots in which she was stationary for five seconds. The blue dots represent the position estimates returned by the RTI system.

riding a stationary bike, etc. For example, an RTI system can be used by doctors and caretakers as a non-invasive way to monitor the eating habits and the level of mobility of an obese person while at home. The movements of a person in different AoIs can be reliably detected by using a finite-state machine [30], in which each state is associated to a different AoI. The temporal sequence of state transitions the and time spent in each AOI measured by the RTI system can be used to extract spatiotemporal activity patterns [44].

\subsection{EvAAL Competition Deployment}

The RTI system described in this chapter participated to the second EvAAL (Evaluating AAL Systems through Competitive Benchmarking) competition 45. in the track on indoor localization and tracking. Figure 12 shows the blueprint of the home in which the experimental evaluation was carried out. In it, the yellow circles represent the RF sensors, the black dots the true position of the person during the test at $1 \mathrm{~s}$ intervals, the red dots the true position of the person at spots in which she was stationary for five seconds, and the blue dots the position estimates returned by the RTI system. The $42 \mathrm{RF}$ sensors were installed by one person in approximately 43 minutes. The position of the nodes was decided before their deployment in order to be able to pre-calculate the inversion matrix $\Pi$ in (14). However, in order to speed up the deployment, the installer positioned the nodes by using the furniture found in the home as reference, i.e., without 
precisely measuring the distances among the nodes. This inevitably introduced an error for some nodes between their real coordinates and the ones used in the computation of $\Pi$. Despite this small errors, the RTI system was able to accurately track the person moving around the home, as shown in Figure 12.

\section{Conclusion}

This chapter explores the use of radio tomographic imaging (RTI) in the context of ambient assisted living (AAL) applications. In RTI, the received signal strength (RSS) measured on the links of a mesh network composed of static wireless transceivers are used to accurately localize and track people without requiring them to wear or carry any sensor or radio device. The presence of a person on the link line, i.e., the straight imaginary line connecting the transmitter and receiver, changes the measured RSS. We show that this change in RSS depends on the fade level of the measured frequency channel and the distance between the two communicating devices. Based on these findings, the spatial impact area of a link is modeled as an ellipse having the foci at the transmitter and receiver. This ellipse is more narrow for shorter links, and wider for longer links. The concept of fade level is used to weight the RSS measurements collected on different frequency channels: the anti-fade channels are weighted more than the deep fade ones, since their RSS measurements are more informative for localization. By applying a regularized least-square approach, we are able to estimate in real-time a discretized image of the change in the propagation field of the monitored area due to the presence a person. The voxel of the RTI image having the highest intensity represents the estimated position of the person.

RSS-based indoor localization becomes even more challenging in highly dynamic domestic environments, since the propagation patterns of the radio signals can be drastically changed even by small environmental modifications. In this chapter, we introduce methods to make the RTI system able to recalibrate on-line and adapt to the environmental changes introduced by the monitored person during her daily activities. We deploy a system for over three months in a typical single floor, single bedroom apartment inhabited by a single person. Experimental results demonstrate that the average localization error is $0.23 \mathrm{~m}$. In addition, the system provides a high localization accuracy over an extended period of time despite several environmental changes introduced by the person during her daily activities, as shown in the video in [43]. The ability of the RTI system to accurately localize the person without requiring her to participate in the localization effort makes this technology a very attractive solution for AAL and elder-care applications.

Acknowledgments. This work is supported by the US National Science Foundation Grants \#0748206 and \#1035565. 


\section{References}

1. Bultitude, R.J.: Measurement, characterization, and modeling of indoor 800/900 $\mathrm{MHz}$ radio channels for digital communications. IEEE Communications 25(6), 512 (1987)

2. Hashemi, H.: A Study of Temporal and Spatial Variations of the Indoor Radio Propagation Channel. IEEE International Symposium on Personal, Indoor and Mobile Radio Communications, 127-134 (1994)

3. Rappaport, T.: Wireless Communications: Principles and Practice, 2nd edn. Prentice Hall PTR, Upper Saddle River (2001)

4. Liberti, J.C., Rappaport, T.S.: A Geometrically Based Model for Line-of-Sight Multipath Radio Channels. In: 46th IEEE Vehicular Technology Conference, vol. 5, pp. 844-848 (1996)

5. Molisch, A.: Wireless Communications, 2nd edn. John Wiley \& Sons Ltd. (2011)

6. Nørklit, O., Andersen, J.B.: Diffuse Channel Model and Experimental Results for Array Antennas in Mobile Environments. IEEE Transactions on Antennas and Propagation 46(6), 834-840 (1998)

7. Hashemi, H.: The indoor radio propagation channel. Proceedings of the IEEE 81(7), 943-968 (1993)

8. Ghaddar, M., Talbi, L., Denidni, T.: Human Body Modelling for Prediction of Effect of People on Indoor Propagation Channel. Electronics Letters 40, 25 (2004)

9. Wilson, J., Patwari, N.: See-Through Walls: Motion Tracking Using VarianceBased Radio Tomography Networks. IEEE Transactions on Mobile Computing 10(5), 612-621 (2011)

10. Woyach, K., Puccinelli, D., Haenggi, M.: Sensorless Sensing in Wireless Networks: Implementation and Measurements. In: 4th International Symposium on Modeling and Optimization in Mobile, Ad Hoc and Wireless Networks (2006)

11. Patwari, N., Agrawal, P.: Effects of Correlated Shadowing: Connectivity, Localization, and RF Tomography. In: IEEE/ACM International Conference on Information Processing in Sensor Networks, pp. 82-93 (2008)

12. Wilson, J., Patwari, N.: Radio Tomographic Imaging With Wireless Networks. IEEE Transactions on Mobile Computing 9(5), 621-632 (2010)

13. Kanso, M.A., Rabbat, M.G.: Compressed RF Tomography for Wireless Sensor Networks: Centralized and Decentralized Approaches. In: Krishnamachari, B., Suri, S., Heinzelman, W., Mitra, U. (eds.) DCOSS 2009. LNCS, vol. 5516, pp. 173-186. Springer, Heidelberg (2009)

14. Chen, X., Edelstein, A., Li, Y., Coates, M., Rabbat, M., Aidong, M.: Sequential Monte Carlo for Simultaneous Passive Device-free Tracking and Sensor Localization Using Received Signal Strength Measurements. In: ACM/IEEE Information Processing in Sensor Networks (2011)

15. Kaltiokallio, O., Bocca, M.: Real-Time Intrusion Detection and Tracking in Indoor Environment Through Distributed RSSI Processing. In: 17th IEEE International Conference on Embedded and Real-Time Computing Systems and Applications, pp. 61-70 (2011)

16. Kaltiokallio, O., Bocca, M., Patwari, N.: Enhancing the Accuracy of Radio Tomographic Imaging Using Channel Diversity. In: 9th IEEE International Conference on Mobile Ad Hoc and Sensor Systems (2012)

17. Zhang, D., Liu, Y., Ni, L.: Rass: A Real-Time, Accurate and Scalable System for Tracking Transceiver-Free Objects. In: IEEE International Conference on Pervasive Computing and Communications, pp, pp. 197-204 (2011) 
18. Patwari, N., Wilson, J.: RF Sensor Networks for Device-Free Localization and Tracking. Proceedings of the IEEE 98(11), 1961-1973 (2010)

19. Li, Q., Stankovic, J.A., Hanson, M.A., Barth, A.T., Lach, J., Zhou, G.: Accurate, Fast Fall Detection Using Gyroscopes and Accelerometer Derived Posture Information. In: 6th International Workshop on Wearable and Implantable Body Sensor Networks, pp. 138-143 (2009)

20. Anderson, D., Keller, J., Skubic, M., Chen, X., He, Z.: Recognizing falls from silhouettes. In: 28th International Conference of the IEEE Engineering in Medicine and Biology Society, pp. 6388-6391 (2006)

21. Bahl, P., Padmanabhan, V.: RADAR: an In-building RF-based User Location and Tracking System. In: 19th Conference of the IEEE Computer and Communication Societies, vol. 2, pp. 775-784 (2000)

22. Youssef, M., Mah, M., Agrawala, A.: Challenges: Device-Free Passive Localization for Wireless Environments. In: ACM International Conference on Mobile Computing and Networking, pp. 222-229 (2007)

23. Barsocchi, P., Lenzi, S., Chessa, S., Giunta, G.: A Novel Approach to Indoor RSSI Localization by Automatic Calibration of the Wireless Propagation Model. In: 69th IEEE Vehicular Technology Conference, pp. 1-5 (2009)

24. Viani, F., Rocca, P., Benedetti, M., Oliveri, G., Massa, A.: Electromagnetic Passive Localization and Tracking of Moving Targets in a WSN-Infrastructured Environment. Inverse Problems 26(7) (2010)

25. Wilson, J., Patwari, N.: A Fade-Level Skew-Laplace Signal Strength Model for Device-Free Localization with Wireless Networks. IEEE Transactions on Mobile Computing 11(6), 947-958 (2012)

26. Baccour, N., Koubâa, A., Mottola, L., Zúñiga, M.A., Youssef, H., Boano, C.A., Alves, M.: Radio link quality estimation in wireless sensor networks: A survey. ACM Transactions on Sensor Networks 8(4), 1-34 (2012)

27. Agrawal, P., Patwari, N.: Correlated Link Shadow Fading in Multi-hop Wireless Networks. IEEE Transsactions on Wireless Communications 8(8), 4024-4036 (2009)

28. Zhao, Y., Patwari, N.: Demo Abstract: Histogram Distance-Based Radio Tomographic Localization. In: IEEE/ACM International Conference on Information Processing in Sensor Networks, pp. 129-130 (2012)

29. Phillips, J.M., Venkatasubramanian, S.: A Gentle Introduction to the Kernel Distance. Technical Report arXiv:1103.1625, Arxiv.org (2011)

30. Kaltiokallio, O., Bocca, M., Patwari, N.: Follow @Grandma: Long-Term DeviceFree Localization for Residential Monitoring. In: 7th IEEE International Workshop on Practical Issues in Building Sensor Network Applications (2012)

31. Zhao, Y., Patwari, N.: Noise Reduction for Variance-Based Device-Free Localization and Tracking. In: 8th IEEE Conference on Sensor, Mesh and Ad Hoc Communications and Networks, pp. 179-187 (2011)

32. Wilson, J., Patwari, N., Vasquez, F.G.: Regularization Methods for Radio Tomographic Imaging. In: Virginia Tech. Wireless Symposium (2009)

33. Kalman, R.E.: A New Approach to Linear Filtering and Prediction Problems. Transactions of the ASME Journal of Basic Engineering (Series D) (82), 35-45 (1960)

34. Thouin, F., Nannuru, S., Coates, M.J.: Multi-target Tracking for Measurement Models with Additive Contributions. In: International Conference on Information Fusion (2011) 
35. Nannuru, S., Li, Y., Zeng, Y., Coates, M., Yang, B.: Radio Frequency Tomography for Passive Indoor Multi-Target Tracking. IEEE Transactions on Mobile Computing (2012), http://doi.ieeecomputersociety.org/10.1109/TMC.2012.190

36. Zheng, Y., Men, A.: Through-Wall Tracking with Radio Tomography Networks Using Foreground Detection. In: IEEE Wireless Communications and Networking Conference (2012)

37. Edelstein, A., Rabbat, M.: Background Subtraction for Online Calibration of RF Sensing Networks. IEEE Transactions on Mobile Computing (accepted September 2012)

38. Texas Instruments.: A USB-Enabled System-on-Chip Solution for 2.4GHz IEEE 802.15.4 and ZigBee Applications, http://www.ti.com/lit/ds/symlink/cc2531.pdf

39. IEEE 802.15.4 Standard Technical Specifications, http://www. ieee802.org/15/pub/TG4Expert.html

40. Texas Instruments.: Small Size $2.4 \mathrm{GHz}$ PCB antenna. www.ti.com/lit/an/swra117d/swra117d.pdf

41. Srinivasan, K., Dutta, P., Tavakoli, A., Levis, P.: Understanding the Causes of Packet Delivery Success and Failure in Dense Wireless Sensor Networks. In: 4th ACM Conference on Embedded Networked Sensor Systems, pp. 419-420 (2006)

42. Xing, G., Sha, M., Huang, J., Zhou, G., Wang, X., Liu, S.: Multichannel Interference Measurement and Modeling in Low-Power Wireless Networks. In: IEEE Real-Time Systems Symposium, pp. 248-257 (2009)

43. Kaltiokallio, O.: Follow @Grandma: Long-term Device-Free Localization for Residential Monitoring (YouTube Video), http://www . youtube.com/watch?v=XuMBRm6S_6g

44. Lymberopoulos, D., Bamis, A., Savvides, A.: Extracting Spatiotemporal Human Activity Patterns in Assisted Living Using a Home Sensor Network. Universal Access in the Information Society 10(2), 125-138 (2011)

45. Second EvAAL (Evaluating AAL Systems through Competitive Benchmarking) Competition, http://evaal aaloa.org/2012/2012-competition 